\title{
CROHN'S DISEASE: A ROLE OF GUT MICROBIOTA AND NOD2 GENE POLYMORPHISMS IN DISEASE PATHOGENESIS
}

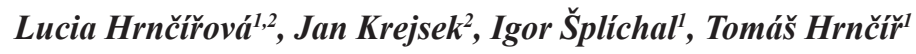

Institute of Microbiology, Academy of Sciences of the Czech Republic, Doly 183, 54922 Nový Hrádek, Czech Republic: Department of Immunology and Gnotobiology ${ }^{1}$; Charles University in Prague, Faculty of Medicine and University Hospital in Hradec Králové, Czech Republic: Department of Clinical Immunology and Allergology²

\begin{abstract}
Summary: Crohn's disease is a chronic immune-mediated intestinal inflammation targeted against a yet incompletely defined subset of commensal gut microbiota and occurs on the background of a genetic predisposition under the influence of environmental factors. Genome-wide association studies have identified about 70 genetic risk loci associated with Crohn's disease. The greatest risk for Crohn's disease represent polymorphisms affecting the CARD15 gene encoding nucleotide-binding oligomerization domain 2 (NOD2) which is an intracellular sensor for muramyl dipeptide, a peptidoglycan constituent of bacterial cell wall. The accumulated evidence suggests that gut microbiota represent an essential, perhaps a central factor in the induction and maintaining of Crohn's disease where dysregulation of normal co-evolved homeostatic relationships between intestinal microbiota and host mucosal immune system leads to intestinal inflammation. Taken together, these findings identify Crohn's disease as a syndrome of overlapping phenotypes that involves variable influences of genetic and environmental factors. A deeper understanding of different genetic abnormalities underlying Crohn's disease together with the identification of beneficial and harmful components of gut microbiota and their interactions are essential conditions for the categorization of Crohn's disease patients, which enable us to design more effective, preferably causative, individually tailored therapy.
\end{abstract}

Key words: Autoimmune diseases; Commensal microflora; Hygiene hypothesis; Inflammatory bowel diseases; Nod2

\section{Crohn's disease}

Crohn's disease (CD), ulcerative colitis, and intermediate colitis are immune-mediated chronic intestinal disorders named together as inflammatory bowel diseases (IBD). IBD is thought to be the result of an overaggressive immune response to a subset of commensal gut microbiota in a genetically susceptible host, with disease initiated by environmental triggers (1). CD is a segmental, transmural inflammation of intestinal wall that can affect any part of gastrointestinal tract from the mouth to the anus with skip areas interspersed between one or more involved areas. Most commonly CD affects terminal ileum, cecum, perianal area, and colon. Histologically, CD manifests itself by a transmural, dense infiltration of lymphocytes and macrophages, the presence of granulomas (in up to $60 \%$ of patients), fissuring ulceration and submucosal fibrosis. CD can cause significant morbidity such as diarrhea, pain, narrowing of the gut lumen leading to strictures and bowel obstruction, abscess formation, and fistulization to skin and internal organs. CD can also be associated with other medical conditions, including arthritis, osteoporosis, eye inflammation, blood clots, liver disease, and skin rashes.

\section{Incidence}

IBD are a public health problem in industrialized countries, where two in 1,000 people are affected. Most patients are young adults. The incidence of IBD has increased greatly in western countries since the Second World War but is beginning to level off. However, the incidence is still rising in low-incidence areas such as Eastern Europe, Asia, and developing countries (2). The highest incidence of CD has been reported in northern Europe, the United Kingdom, North America $(8-14 / 100,000)$ and New Zealand; the most affected is Canterbury County, New Zealand with incidence $16.5 / 100,000$ people (3). The prevalence of CD in the West is $120-200 / 100,000$ persons (3-5). The incidence is about 1-3 per 100,000 in southern Europe, South Africa, and Australia, and is even lower, less than 1 per 100,000, in Asia and South America. CD is more prevalent in whites than in African Americans and Asians. In the United States, Europe, 
and South Africa, Crohn's disease is 2-4 times more common among Jewish people of Ashkenazi origin than among other ethnic or social groups (6).

\section{Etiology}

The etiology of IBD has been extensively studied. However, causative factors in disease pathology are not yet fully understood. IBD is thought to result from the interaction between genetic and environmental factors that influence the composition of normal commensal gut microbiota to trigger an inappropriate mucosal immune response (7).

The mucosa of gastrointestinal tract with surface of approximately $200 \mathrm{~m}^{2}$ forms a platform where large quantities of antigenic, mitogenic and toxic stimuli present in food together with resident commensal microbiota interact with cells of the human body. These two compartments are separated by physical barriers formed by epithelial layer single layer of interconnected, polarized epithelial cells, reinforced by tight junctions, and a basement membrane, which separates it from the connective and supporting tissue, by chemical barriers - mucus and humoral factors, i.e. secretory immunoglobulin A and antimicrobial peptides, and biological barriers, which ensure microbiota as a compact ecologic community. But these two worlds are not ultimately divided, in fact in the gut there is a complex network of sensing and regulatory signaling cascades that is essential for proper activation together with a timely inactivation of the pathway $(8,99)$.

A characteristic feature of the immune system in mucosa is mucosal tolerance, the ability of discriminating between potentially pathogenic microorganisms and harmless antigens, it has developed a great redundancy of various mechanisms that ensure essential defense functions and simultaneously the prevention of immune system stimulation to food antigens, environmental allergens and components of microbiota. The immune system has two, broadly cooperative components. The first line of defense, the innate nonspecific immune system, which comprises a large number of cell populations present in mucosa and mucosa-associated lymphoid tissue and relies on humoral factors and germline-encoded pattern recognition receptors, promotes the immediate detection and rapid destruction of microorganisms. The cells of innate immunity also produce factors essential for subsequent initiation of specific immunity. Tight control of innate immunity is critical to mucosal homeostasis in the intestine. An adaptive, antigen-specific immune system arises as a consequence of antigen exposure. Adaptive immunity is initiated when antigen-presenting cells, primarily dendritic cells, present antigen to lymphocytes in inductive immune compartments, such as lymph nodes and Peyer's patches. Naive T cells mature to effector T lymphocyte: $\mathrm{T}_{\mathrm{C}}, \mathrm{T}_{\mathrm{H}} 1, \mathrm{~T}_{\mathrm{H}} 2, \mathrm{~T}_{\mathrm{H}} 17$ or $\mathrm{T}_{\text {reg }}$ lineages depending on additional signals and cytokine milieu. Intraepithelial lymphocytes serve to regulate intestinal homeostasis, maintain epithelial barrier function, respond to infection and regulate adaptive and innate immune responses (9). B cells mature in $\mathrm{T}$ cell dependent and $\mathrm{T}$ cell independent routes into plasma cells and commit predominantly to IgA production by class-switch recombination $(8,10,11,99)$.

The microenvironment of the gut is mainly tolerogenic, the dominant mucosal immune response mechanisms are those that dampen the immune and inflammatory responses and limit inflammation that could injure the mucosal layer. Mucosal tolerance to microbiota is a fundamental mechanism of maintaining intestinal homeostasis. The major mechanisms underlying immunologic tolerance generally include deletion of antigen-reactive $\mathrm{T}$ cells, clonal anergy of antigen-reactive T cells, and induction of antigen-specific regulatory T cells. Diminished T-cell-mediated responses efficiently suppress the otherwise unavoidable overstimulation of the immune system. The concomitant induction of humoral immune responses in the mucosal compartment and T-cell unresponsiveness in the systemic compartment are two mutually complementary mechanisms which in concert achieve fundamental defense principles (8).

During the last decade new metagenomic approaches were used to analyze the composition of microbiota and its metagenome. The interactions between the gut microbiota and the host are analyzed in fuctional studies. To investigate the role of microbiota in the development of immune system (12), the pathogenesis of inflammatory diseases (13, $14)$, autoimmune diseases and cancer (15) gnotobiological approaches are exploited (11).

\section{Environmental factors}

Environmental risk factors involved in IBD include factors that have an influence on the composition of gut microbiota (i.e. maternal exposure, breastfeeding, diet, antibiotics, infections), and factors that affect the mucosal immune system (i.e. smoking, non-steroidal anti-inflammatory drugs, oral contraceptive pills, stress, vaccination, intestinal permeability, and appendectomy) (1, 16, 17). Improved sanitation and hygiene along with decreased exposure to enteric organisms during early childhood, may lead to a greater susceptibility of developing an inappropriate immunological response upon exposure to new antigens later in life $(18,19)$. The finding is supported by epidemiological data and forms the basis of the so-called "hygiene hypothesis".

\section{Genetic factors}

An important role of genetic factors in IBD was first suggested by epidemiological studies showing familial aggregation of IBD and by twin studies. Monozygotic twin studies show the concordance rate for disease being 40-60\% (20).

Genome-wide association studies (GWAS) have identified single nucleotide polymorphisms (SNP) in 71 genes that are associated with $\mathrm{CD}(21)$. Functionally, these genetic 
risks can be characterized broadly as abnormalities in innate immune responses, immunoregulation, or mucosal barrier function, namely, Nod2-dependent innate immunity, barrier function, epithelial restitution, microbial defense, innate immune regulation, reactive oxygen species (ROS) generation, autophagy, the inflammasome pathway, regulation of adaptive immunity, interleukin-23 (IL-23)-IL-17 circuitry, endoplasmic reticulum stress, and metabolic pathways associated with cellular homeostasis (22).

In particular, Nod 2 on chromosome 16 was the first gene associated with susceptibility to CD (23). Among the three main SNPs identified in Nod2 (R702W, G908R, L1007fs), the L1007fs (frame shift stop-codon mutation that leads to a partial deletion of the terminal LRR of the protein) displays the strongest association with CD (23-25) and 1007fs homozygous patients demonstrate much severe disease phenotype than other patients with $C D$ with an early disease onset and long-segment ileal stenosis and entero-enteral fistulas. They frequently need a surgical intervention and have a high risk of restenosis. This mutation is also associated with gastroduodenal involvement (26-28). In addition to CD, three missense mutations (R334W, R334QQ an L469F) in the nucleotide-binding domain of Nod2 confer susceptibility to another granulomatous disorder affecting the eyes, skin, and joints known as Blau syndrome (29) and early onset sarcoidosis (30).

Between $30 \%$ and $50 \%$ of CD patients in the Western hemisphere carry at least one mutated Nod2 allele. Individuals who carry two of these mutated Nod 2 alleles have a $20-40$-fold increased risk of developing CD $(23,24)$. The patients with double-dose mutations are characterized by a younger age at onset, a more frequent stricturing phenotype, and a less frequent colonic involvement than are seen in those patients who had no mutation (31). However, normal individuals might have Nod 2 mutations on both chromosomes in the absence of disease (32) and also mice deficient in Nod2 do not develop CD spontaneously (33). In some populations the carrier rate in healthy controls of CARD15 mutations associated with Crohn's disease is $20 \%$ (34). None of the three mutations in the Nod2 gene was found in 483 Japanese patients with CD (35) and also were not to be associated with CD in Chinese (36) and Korean (37) patients. It is of great interest and relevance to note that the three LRR domain mutations are also associated with graft-versus-host disease (38).

\section{Nod2 structure and function}

Nod2 (nucleotide-binding oligomerization domain 2) is an important host defense and regulatory factor. It is a germ line-encoded pattern-recognition receptor, which belongs to the NLR (nucleotide-binding domain, leucine rich-repeat containing) family. Pattern recognition receptors of the innate immune system cells and epithelial cells, including Toll-like receptors (TLRs), NLRs, C-type lectins, and retinoic acid-inducible gene I-like receptors, sense microbial and danger motifs and are required for the activation and shaping of the adaptive and regulatory immune responses, mucosal regeneration and tissue repair (22).

Nod2 contains two tandem caspase recruitment domains (CARD1 and 2) on its N-terminal side, a central nucleotide-binding domain (NBD), and leucine-rich repeat (LRR) domain on its C-terminus. The P-loop containing the ATP-binding motif is located in the NBD domain and allows conformational change of the molecule. The LRR domain has receptor function and is also responsible for cell membrane association of Nod2 (39). The three main CD- and GvHD-associated SNPs (R702W, G908R and 1007fs) are in LRR domain and the Blau syndrome-associated mutations (R334W, R334Q and L469F) are in the NBD domain.

Nod2 recognizes muramyl dipeptide (MDP), a component of bacterial cell wall peptidoglycan, and viral ssRNA (40). Nod2 is found both in the cytoplasm and in association with the cell membrane through two amino acids in LRR domain, membrane association is necessary for Nod2 stimulation (39). Nod2 is expressed in myeloid cells such as monocytes (41), macrophages, dendritic cells, Paneth cells, and non-Paneth intestinal epithelial cells (42-45) and in T lymphocytes (46) and is recognized as an important mediator of inflammatory response largely dependent on NF- $\kappa$ B activation. Nod2 stimulation can lead to Th2 driven adaptive response $(47,48)$ or Th1 and Th17 responses $(47$, 49) depending on cytokine milieu.

Binding of MDP to Nod 2 causes conformational change of Nod2 structure, which results in an unfolding of the molecule, followed by oligomerization, and exposure of the CARD domain. The CARD domain of Nod2 binds to the CARD domain of RIP2, a threonine-serine kinase, and enables RIP2 to undergo polyubiquitination $(50,51)$. The pattern of polyubiquitination leads to mitogen-activated protein (MAP) kinase pathway (ERK 1/2, p38 and JNK) and/or $\mathrm{NF}-\kappa \mathrm{B}$ activation and the bifurcation in signaling between the NF- $\kappa \mathrm{B}$ and ERK versus p38 and JNK pathways might involve differential recruitment of adaptor proteins that direct signaling towards specific inflammatory responses (52). Bid, a BCL2 family member, is required for downstream Nod2 activation of NF- $\kappa B$, which confirms Nod 2 cross talk with apoptosis proteins (53). Finally, Nod2 may also bind to one or more inflammasome proteins that contain CARD domains such as NLRP-1 or NLRP-3 and thereby participate in IL-1 $\beta$ secretion (54).

Binding of viral ssRNA to Nod2 leads to translocation of Nod2 to mitochondria and interaction with mitochondrial antiviral-signaling protein (MAVS) and activation of NF- $\kappa B$ in the RIP2-independent manner, and interferon regulatory factor-3, and production of interferon $\beta$ (40).

Nod2 signaling is necessary for bacterial autophagy, the breakdown of invading bacterium by forming double-membrane vacuoles that ultimately fuse with lysosomes to eliminate proteins arising from cellular stress responses (55). Nod2 directly interacts with autophagy related 16-like 1 protein (ATG16L1) to recruit it to the plasma membrane at the 
entry foci of bacteria and thus facilitates the formation of an autophagosome around the invading bacterium (56). Nod2 stimulation by MDP leads to upregulation of MHC class II surface expression and fusion of autophagosomes with specific MHC class II compartments (57). Autophagy has been reported to inhibit the generation of reactive oxygen species, which have been shown to trigger the activation of NLRP-3 inflammasomes (58). Autophagy may also inhibit pyroptosis, a highly inflammatory form of caspase-1-dependent cell death that has been observed in myeloid cells infected with intracellular pathogens (59).

Nod2 directly influences the composition of gut microbiota by regulating the production of a subgroup of intestinal antimicrobial peptides, known as cryptdins produced by Paneth cells in intestinal crypts (33). Impairment of Nod2 function leads to deficient production of $\alpha$-defensins by intestinal crypt cells and thereby defective killing of bacteria and increased burden of commensal and pathogenic bacteria in the terminal ileum of Nod2-deficient mice that increases their invasion and contributes to the deeper, often transmural inflammation observed with ileal CD $(60,61)$.

Nod2 mediates an immune tolerance to bacterial products in human and mouse. In vitro, Nod 2 stimulation by MDP lead to release of pro-inflammatory NF- $\kappa B$-dependent cytokines (such as IL-1 $\beta$, TNF- $\alpha$ and IL-6), as well as secretion of IL-23 (which promotes Th17 differentiation) after costimulation with MDP and TLR-2 ligands and deletion of Nod2 in murine cells or loss of Nod 2 function mutations in the LRR ligand recognition domains of Nod2 from human donors lead to a decrease in NF- $\kappa$ B activation, MAPK signaling and pro-inflammatory cytokine secretion $(33,41)$. Whereas in in vivo animal models of inflammation the role of NOD2 deficiency has varied, with some models showing deficient mice having increased inflammation $(60,62)$ and other models showing deficient mice having decreased inflammation $(33,63-65)$. These differences are related to the "chronicity" of the infection: during the early stages of infection Nod 2 is a positive regulator of inflammation, however, after a period of sustained stimulation, the role of Nod2 switches and it becomes a negative regulator of inflammation, potentially via the induction of tolerance to further microbial stimulation through either Nod2 itself or other microbial receptors, such as TLR2 and TLR4 (65-67). This is dependent on IRF4 in mice and humans and downregulation of the IRAK-1 kinase and perhaps upregulation of IL-1R-associated kinase M (IRAK-M) in humans and also on early secretion of IL-10, TGF- $\beta$, IL-1Ra (10). Nod2-dependent release of IL-10 after MDP stimulation has been demonstrated to be specific to humans and is impaired in L1007fs cells (68). Resistance, which clears the invading organisms, and tolerance, which diminishes the negative effects of the host immune response, have been recognized as separate defense strategies in microbial defense.

During allogeneic bone marrow transplantation lack of Nod 2 regulatory function in dendritic cells leads to enhanced proliferation and activation of allogeneic donor T cells pre- sumably under the influence of endogenous TLR ligands, which results in target organ damage in graft versus host disease (GvHD) (69).

Nod2 is intrinsically required for $\mathrm{T}$ cell function. TCR and CD28 signaling triggers the activation of Nod2, which then interacts with NF- $\mathrm{KB}$ inducing kinase (NIK) and c-Rel to form a complex that promotes c-Rel nuclear accumulation (46). C-Rel mediated IL-2 production positively regulates $\mathrm{T}$ cell activation and differentiation and plays a crucial role in $\mathrm{T}$ cell priming for IFN- $\gamma$ production (70). Studies have demonstrated that c-Rel that is activated in response to TCR and CD28 triggering binds to regulatory element present in the forkhead box P3 (Foxp3) promoter region to facilitate Foxp3 expression and to promote T-regulatory (Treg) cell differentiation an thus may contribute to dysregulated immune response in Nod2-deficient host (69).

Nod2-deficient mice exhibit a hyperplasia and a hypertrophy of the Peyer's patches. The Th1 immune activation and increased levels of mucosal IFN- $\gamma$ and TNF- $\alpha$ play a key role in the disruption of the epithelial barrier integrity of Peyer's patches of Nod2-deficient mice and lead to increased transcellular permeability and bacterial translocation in Peyer's patches (71).

Mutations in the Nod 2 gene are strong genetic risk factors for ileal CD; however, the mechanism by which these mutations predispose to increased intestinal inflammation remains a subject of controversy. They include a role of Nod2 in the induction of defensins production, impaired autophagy and antigen presentation, mediating tolerance by attenuating inflammatory responses initiated by other receptors and secretion of anti-inflammatory cytokines, inhibiting processing and secretion of IL-1 $\beta$ and pyroptosis and impaired $\mathrm{T}$ cell differentiation and proliferation.

\section{Gut microbiota}

Analysis of mouse models has revealed at least two major courses of disease: dysbiosis characterized by the depletion or alteration of commensal microbiota and chronic pathogen infection. Inflammation might arise from a lack of tolerance to antigens present in autologous microflora or from transient infection by traditional enteric pathogens which might break the mucosal barrier and activate pathogenic immune responses that are subsequently perpetuated by commensal enteric antigens in genetically susceptible host who is unable to repair epithelial breaches or downregulate the inflammatory response (72).

The essential role for the commensal microbiota as antigenic stimuli of effector immune responses in chronic intestinal inflammation is broadly accepted. Although it is not clear whether dysbiosis can cause IBD in humans or is a consequence of acute infection or host inflammatory response, several lines of evidence indicate that dysbiosis consisting of a decrease in beneficial bacteria and their metabolic end-products together with an increase of detrimental bacterial populations and their toxic metabolites drives ac- 
tivated inflammatory cascades leading to CD in genetically susceptible host $(1,73)$. Dysbiosis in CD is characterized by decreased microbial diversity in major phyla, such as Firmicutes and Bacteroidetes, altered ratio of beneficial and aggressive bacterial species and higher numbers of mucosa-associated bacteria than have healthy individuals (72).

Patients with ileal and colonic CD have significantly reduced concentrations of core commensals belonging to the Clostridiales order, such as Faecalibacterium prausnitzii and Roseburia (74). These genera are potent sources of short-chain fatty acids (75) and clostridial groups IV and XIVa promote the accumulation of Foxp $3^{+}$Treg cells in the mouse colon (76). Decreased number of $F$. prausnitzii in resected ileal CD mucosa is a predictive marker of postoperative ileal CD (77).

Bacterial species that are consistently increased in CD patients include Escherichia coli, specifically the B2 and D phylotypic groups and adherent/invasive strains (AIEC) associated with ileal CD $(78,79)$. AIEC adhere to the ileal mucosa through binding to molecules, which are overexpressed during $\mathrm{CD}(80,81)$. AIEC can also disrupt the integrity of polarized cell monolayer, breach the intestinal barrier and penetrate into the gastrointestinal epithelium (82). By expressing long polar fimbriae, the bacteria interact with mouse and human Peyer's patches and translocate across microfold cells monolayers (83). AIEC are also able to survive and replicate extensively within a large, phagolysosome-like vacuole in macrophages (84) and AIEC-infected macrophages aggregate and fuse to form multinucleated giant cells in vitro (85).

An etiological role for other intracellular opportunistic pathogen, Mycobacterium avium subspecies paratuberculosis or measles virus is not widely supported. The higher prevalence of intracellular pathogens in the tissue of CD patients might arise from an inability of the dysfunctional innate immune system to control persistent infection by intracellular bacteria - possibly opportunistic pathogens in intestinal mucosa. However, CD-associated microbes could promote disease in genetically susceptible hosts with defects in innate immune system-mediated killing of microbes, mucosal barrier functions, or immunoregulation.

\section{Pathogenesis of Crohn's disease}

The consensus view of the cause of CD include genetic predisposition, an abnormal immune response to components of the normal gut microflora, and an environmental trigger and define $\mathrm{CD}$ as the genetically supported inappropriately aggressive Th1 and/or Th17 immune response to a subset of commensal intestinal bacteria, initiated and reactivated by transient infectious or environmental triggers (72). This excessive response can arise from an abnormal reactivity of the mucosal immune system to mucosal antigens or from increased exposure of luminal bacteria to normal mucosal immune system (54). Initial nonspecific immune response becomes chronic because of the constant drive of commensal microbial antigens caused by genetic defects of epithelial barrier integrity, bacterial handling and immunoregulation (72).

Th1-mediated immune responses are typically triggered by an intracellular pathogen antigen presentation on MHC class II by antigen-presenting cell (APC) with costimulatory signals in the presence of IL-12 and is aimed to localize the infectious agent, promote intracellular killing or induce the differentiation of cytotoxic T lymphocytes. The hallmark of a Th1 response is a granuloma. Th1 cells under the control of a master transcriptional regulator Tbet produce pro-inflammatory cytokines IFN $\gamma, \mathrm{TNF} \alpha$, and IL-2 (86). These cytokines act on local cell populations to promote intracellular killing, enhance recruitment of other inflammatory cells, enhance secretion of chemoattractant cytokines, and promote local tissue destruction. TNF- $\alpha$ is a central mediator of the intestinal inflammation and is produced by macrophages and Th17 cells (87). TNF- $\alpha$ in turn induces expression of IL-1 $\beta$ and IL-6, both of which are also upregulated in serum of patients with IBD (88). IL-6 is an important factor for the synthesis of acute phase proteins, controls proliferation and resistance of resting $\mathrm{T}$ cells against apoptosis, activates Th2 cytokine production in CD4+ T lymphocytes and together with TGF- $\beta$ induces the generation of Th17 cells while it inhibits differentiation of regulatory $\mathrm{T}$ cells. In particular, IL-6 trans-signaling appears to promote the maintenance of IL-17-secreting T lymphocytes in inflamed tissue (89).

Development of Th17 cells requires TGF- $\beta$ and IL-6 (or IL-21) and is independent of the Th1 pathway. Th17 cells demonstrate substantial developmental plasticity after their commitment to the Th17 program: antigen activated naïve CD4+ T cells respond to TGF $\beta$ to transiently co-express ROR $\gamma$ t and Foxp3, but differentiate into either Th17 cells or induced regulatory $\mathrm{T}$ cells (iTreg) depending on the dominance of IL-6 or all-trans retinoic acid, respectively. Depending on the balance of TGF- $\beta$, IL-23, and IL-12, Th17 precursor diverges into progeny that express high levels of IL-17A and IL-17F (TGF $\beta$ dominance), IL-17A alone, IL22 and IFN $\gamma$ (IL-23 dominance) or suppress IL-17A and IL-17F to express a Th1 pattern of cytokines dominated by IFN $\gamma$ (IL-12 dominance) (90). IL-23 is induced by PRR stimulation and is constitutively expressed in a small population of ileal dendritic cells. During CD, CD14+ intestinal macrophages secrete large amounts of IL-23 (91). IL-23 promotes a wide range of pathological responses in the intestine, mediated either by excessive innate immune activation or by enhancement of Th1 and Th17 responses, including enhanced proliferation of effector T cells, reduced differentiation of Foxp3 + Treg cells and the emergence of IL-17+IFN- $\gamma+$ CD4+ T cells (92). Th17 cells produce several cytokines, including IL-17A, IL-17F, IL-21 and IL-22 (93). IL-17A an IL17F have pro-inflammatory effects in the gut (94). IL-22 mediates either tissue-protective or pathogenic functions, depending on the absence or presence of IL-17A, respectively (95). Depending on the cytokine milieu and tissue in which it is expressed, IL-22 can regulate 
the expression of genes encoding molecules associated with inflammation, repair or chemotaxis or the expression of antimicrobial peptides, which can orchestrate host-protective immunity, tissue inflammation, repair or homeostasis (96). CD patients have increased levels of IL-22 which correlate with increased disease activity and susceptibility-associated IL23R polymorphisms (97).

Th17 response is both permissive and inhibitory of the Th1 response, probably at different phases of the inflammatory cycle, so it is hypothesized that in Crohn's disease Th17 response may be more important in the regulation of the inflammation than in its induction: IL-23 inhibits Treg cell generation and counteracts the inhibitory effect of Treg cells on both Th1 and Th17 proinflammatory responses and IL-17 inhibits generation of IFN $\gamma$-producing cells (98). In the proposed model of pathogenesis of Crohn's disease the intestinal inflammation consists of innumerable microenvironments, each exhibiting a progression of inflammatory patterns. In the initial and most intense phase of the inflammation Th1 responses predominate, at this point, production of IL-23 in a nascent Th17 response inhibits regulatory T-cells generation and feeds the inflammation. In a later phase, a mixed T-cell response prevails in which the Th1 response is still predominant but is now moderated by a Th17 response producing both IL-17 (which inhibits IFN- $\gamma$ T cells) and IL-22 (98).

\section{Conclusion}

Since the first description of CD in 1932, the understanding of the etiology and pathogenesis of disease underwent a substantial progress. GWAS have identified numerous genomic regions containing CD-risk factors, revealing several features of the genetic architecture of CD. Furthermore, the importance of multiple environmental factors in the induction and maintaining of CD was established. The accumulated evidence suggests that gut microbiota represent an essential, perhaps a central factor in the development of CD where dysregulation of normal co-evolved homeostatic relationships between intestinal microbiota and host mucosal immune system leads to intestinal inflammation. Taken together, these findings identify CD as a syndrome of overlapping phenotypes that involves variable influences of genetic and environmental factors. A deeper understanding of different genetic abnormalities underlying CD together with the identification of beneficial and harmful components of gut microbiota and their interactions are essential conditions for the categorization of CD patients, which enable us to design more effective, preferably causative, individually tailored therapy.

\section{Acknowledgements}

This work was supported by Charles University in Prague, Faculty of Medicine in Hradec Králové, Czech Republic, project "PRVOUK" P37/10, by grant from the Grant Agency of Charles University in Prague (No. 906613) and by grants from the Academy of Sciences of the Czech Republic (No. M200201207), IGA Ministry of Health of the Czech Republic (No. NT14017), the Grant Agency of Czech Republic (P303/12/0535) and Institutional Research Concept (RVO: 61388971).

\section{References}

1. Sartor RB. Genetics and environmental interactions shape the intestinal microbiome to promote inflammatory bowel disease versus mucosal homeostasis. Gastroenterology 2010; 139(6): 1816-9.

2. Colombel JF, et al. Epidemiology and risk factors of inflammatory bowel diseases. Bulletin de l'Academie nationale de medecine 2007; 191(6): 1105-18; discussion $1118-23$.

3. Cosnes J, et al. Epidemiology and natural history of inflammatory bowel diseases. Gastroenterology 2011; 140(6): 1785-94.

4. Hiatt RA, Kaufman L. Epidemiology of inflammatory bowel disease in a defined northern California population. West J Med 1988; 149(5): 541-6.

5. Moum B, et al. Incidence of Crohn's disease in four counties in southeastern Norway, 1990-93. A prospective population-based study. The Inflammatory Bowel South-Eastern Norway (IBSEN) Study Group of Gastroenterologists. Scand J Gastroenterol 1996; 31(4): 355-61.

6. Baumgart DC, et al. IBD Around the world: comparing the epidemiology, diagnosis, and treatment: proceedings of the World Digestive Health Day 2010 Inflammatory Bowel Disease Task Force meeting. Inflammatory bowel diseases 2011; 17(2): 639-44.

7. Molodecky NA, Kaplan GG. Environmental risk factors for inflammatory bowel disease. Gastroenterol Hepatol (NY) 2010; 6(5): 339-46.

8. Tlaskalova-Hogenova $\mathrm{H}$, et al. Interaction of mucosal microbiota with the innate immune system. Scand J Immunol 2005; 62(Suppl 1): 106-13.

9. Weiner HL, et al. Oral tolerance. Immunol Rev 2011; 241(1): 241-59.

10. Khor B, Gardet A, Xavier RJ. Genetics and pathogenesis of inflammatory bowel disease. Nature 2011; 474(7351): 307-17.

11. Tlaskalova-Hogenova $\mathrm{H}$, et al. The role of gut microbiota (commensal bacteria) and the mucosal barrier in the pathogenesis of inflammatory and autoimmune diseases and cancer: contribution of germ-free and gnotobiotic animal models of human diseases. Cellular \& molecular immunology 2011; 8(2): 110-20.

12. Hrncir T, et al. Gut microbiota and lipopolysaccharide content of the diet influence development of regulatory T cells: studies in germ-free mice. BMC Immunol 2008; 9: 65.

13. Stepankova R, et al. Segmented filamentous bacteria in a defined bacterial cocktail induce intestinal inflammation in SCID mice reconstituted with CD45RBhigh CD4+ T cells. Inflammatory bowel diseases 2007; 13(10): 1202-11.

14. Singh B, et al. Control of intestinal inflammation by regulatory $\mathrm{T}$ cells. Immunol Rev 2001; 182: 190-200.

15. Klimesova K, et al. Altered gut microbiota promotes colitis-associated cancer in IL-1 receptor-associated kinase M-deficient mice. Inflammatory bowel diseases 2013; 19(6): 1266-77.

16. Danese S, Sans M, Fiocchi C. Inflammatory bowel disease: the role of environmental factors. Autoimmunity reviews 2004; 3(5): 394-400

17. Cadwell K, et al. Virus-plus-susceptibility gene interaction determines Crohn's disease gene Atg16L1 phenotypes in intestine. Cell 2010; 141(7): 1135-45.

18. Shanahan F, Bernstein CN. The evolving epidemiology of inflammatory bowel disease. Curr Opin Gastroenterol 2009; 25(4): 301-5

19. Gent AE, et al. Inflammatory bowel disease and domestic hygiene in infancy. Lancet 1994; 343(8900): 766-7.

20. Halme L, et al. Family and twin studies in inflammatory bowel disease. World journal of gastroenterology 2006; 12(23): 3668-72.

21. Franke A, et al. Genome-wide meta-analysis increases to 71 the number of confirmed Crohn's disease susceptibility loci. Nat Genet 2010; 42(12): 1118-25.

22. Saleh M, Elson CO. Experimental inflammatory bowel disease: insights into the host-microbiota dialog. Immunity 2011; 34(3): 293-302.

23. Hugot JP, et al. Association of NOD2 leucine-rich repeat variants with susceptibility to Crohn's disease. Nature 2001; 411(6837): 599-603

24. Ogura $Y$, et al. A frameshift mutation in NOD2 associated with susceptibility to Crohn's disease. Nature 2001; 411(6837): 603-6.

25. Inohara $\mathrm{N}$, et al. Host recognition of bacterial muramyl dipeptide mediated through NOD2. Implications for Crohn's disease. The Journal of biological chemistry 2003; 278(8): 5509-12.

26. Seiderer J, et al. Homozygosity for the CARD15 frameshift mutation $1007 \mathrm{fs}$ is predictive of early onset of Crohn's disease with ileal stenosis, entero-enteral fistulas, and frequent need for surgical intervention with high risk of re-stenosis. Scand J Gastroenterol 2006; 41(12): 1421-32.

27. Seiderer J, et al. Predictive value of the CARD15 variant $1007 \mathrm{fs}$ for the diagnosis of intestinal stenoses and the need for surgery in Crohn's disease in clinical practice: results of a prospective study. Inflammatory bowel diseases 2006; 12(12): 1114-21. 
28. Mardini HE, et al. Gastroduodenal Crohn's disease is associated with NOD2 CARD15 gene polymorphisms, particularly L1007P homozygosity. Dig Dis Sc 2005; 50(12): 2316-22.

29. Miceli-Richard C, et al. CARD15 mutations in Blau syndrome. Nature genetics 2001; 29(1): 19-20.

30. Kanazawa N, et al. Early-onset sarcoidosis and CARD15 mutations with constitutive nuclear factor-kappaB activation: common genetic etiology with Blau syndrome. Blood 2005; 105(3): 1195-7.

31. Lesage S, et al. CARD15/NOD2 mutational analysis and genotype-phenotype correlation in 612 patients with inflammatory bowel disease. American journal of human genetics 2002; 70(4): 845-57.

32. Linde K, et al. Card 15 and Crohn's disease: healthy homozygous carriers of the 3020insC frameshift mutation. The American journal of gastroenterology 2003; 98(3): 613-7.

33. Kobayashi KS, et al. Nod2-dependent regulation of innate and adaptive immunity in the intestinal tract. Science 2005; 307(5710): 731-4.

34. Esters N, et al. Transmission of CARD15 (NOD2) variants within families of patients with inflammatory bowel disease. The American journal of gastroenterology 2004; 99(2): 299-305.

35. Yamazaki K, et al. Absence of mutation in the NOD2/CARD15 gene among 483 Japanese patients with Crohn's disease. Journal of human genetics 2002; 47(9): 469-72.

36. Guo QS, et al. NOD2 3020insC frameshift mutation is not associated with inflammatory bowel disease in Chinese patients of Han nationality. World journal of gastroenterology : WJG 2004; 10(7): 1069-71.

37. Jang JY, et al. Lack of common NOD2 mutations in Korean pediatric patients with inflammatory bowel disease. Pediatrics international: official journal of the Japan Pediatric Society 2010; 52(6): 888-9.

38. Holler E, et al. Both donor and recipient NOD2/CARD15 mutations associate with transplant-related mortality and GvHD following allogeneic stem cell transplantation. Blood 2004; 104(3): 889-94.

39. Barnich N, et al. Membrane recruitment of NOD2 in intestinal epithelial cells is essential for nuclear factor- $\{\mathrm{kappa}\} \mathrm{B}$ activation in muramyl dipeptide recognition. J Cell Biol 2005; 170(1): 21-6.

40. Sabbah A, et al. Activation of innate immune antiviral responses by Nod2. Nature immunology 2009; 10(10): 1073-80.

41. Ogura Y, et al. Nod2, a Nod1/Apaf-1 family member that is restricted to monocytes and activates NF-kappaB. The Journal of biological chemistry 2001; 276(7): 4812-8.

42. Hisamatsu T, et al. CARD15/NOD2 functions as an antibacterial factor in human intestinal epithelial cells. Gastroenterology 2003; 124(4): 993-1000.

43. Xavier RJ and Podolsky DK, Unravelling the pathogenesis of inflammatory bowel disease. Nature 2007; 448(7152): 427-34

44. Barrett JC, et al. Genome-wide association defines more than 30 distinct susceptibility loci for Crohn's disease. Nature genetics 2008; 40(8): 955-62.

45. Coombes JL, Powrie F. Dendritic cells in intestinal immune regulation. Nature reviews. Immunology 2008; 8(6): 435-46.

46. Shaw $\mathrm{MH}$, et al. $\mathrm{T}$ cell-intrinsic role of Nod 2 in promoting type 1 immunity to Toxoplasma gondii. Nat Immunol 2009; 10(12): 1267-74.

47. Fritz JH, et al. Nod1-mediated innate immune recognition of peptidoglycan contributes to the onset of adaptive immunity. Immunity 2007 ; 26(4): 445-59.

48. Magalhaes JG, et al. Nod2-dependent Th2 polarization of antigen-specific immunity. Journal of immunology 2008; 181(11): 7925-35.

49. van Beelen AJ, et al. Stimulation of the intracellular bacterial sensor NOD2 programs dendritic cells to promote interleukin-17 production in human memory $\mathrm{T}$ cells. Immunity 2007; 27(4): 660-9.

50. Hasegawa M, et al. A critical role of RICK/RIP2 polyubiquitination in Nod-induced NF-kappaB activation. EMBO J 2008; 27(2): 373-83.

51. Abbott DW, et al. The Crohn's disease protein, NOD2, requires RIP2 in order to in duce ubiquitinylation of a novel site on NEMO. Curr Biol 2004; 14(24): 2217-27.

52. Tao M, et al. ITCH K63-ubiquitinates the NOD2 binding protein, RIP2, to influence inflammatory signaling pathways. Curr Biol 2009; 19(15): 1255-63.

53. Yeretssian G, et al. Non-apoptotic role of BID in inflammation and innate immunity. Nature 2011; 474(7349): 96-9.

54. Strober W, Watanabe T. NOD2, an intracellular innate immune sensor involved in host defense and Crohn's disease. Mucosal immunology 2011.

55. Mizushima N, et al. Autophagy fights disease through cellular self-digestion. Nature 2008; 451(7182): 1069-75.

56. Travassos LH, et al. Nod1 and Nod2 direct autophagy by recruiting ATG16L1 to the plasma membrane at the site of bacterial entry. Nature immunology 2010; 11(1): 55-62.

57. Cooney R, et al. NOD2 stimulation induces autophagy in dendritic cells influencing bacterial handling and antigen presentation. Nat Med 2010; 16(1): 90-7.

58. Zhou R, et al. A role for mitochondria in NLRP3 inflammasome activation. Nature 2011; 469(7329): 221-5.

59. Maloy KJ and Powrie F, Intestinal homeostasis and its breakdown in inflammatory bowel disease. Nature 2011; 474(7351): 298-306.

60. Biswas A, et al. Induction and rescue of Nod2-dependent Th1-driven granulomatous inflammation of the ileum. Proceedings of the National Academy of Sciences of the United States of America 2010; 107(33): 14739-44.
61. Petnicki-Ocwieja T, et al. Nod2 is required for the regulation of commensal microbiota in the intestine. Proc Natl Acad Sci U S A 2009; 106(37): 15813-8.

62. Watanabe $\mathrm{T}$, et al. Nucleotide binding oligomerization domain 2 deficiency leads to dysregulated TLR2 signaling and induction of antigen-specific colitis. Immunity 2006; 25(3): 473-85.

63. Frutuoso MS, et al. The pattern recognition receptors Nod 1 and Nod 2 account for neutrophil recruitment to the lungs of mice infected with Legionella pneumophila. Microbes Infect 2010; 12(11): 819-27.

64. Geddes K, et al. Nod1 and Nod2 regulation of inflammation in the Salmonella colitis model. Infection and immunity 2010; 78(12): 5107-15.

65. Petnicki-Ocwieja T, et al. Nod2 suppresses Borrelia burgdorferi mediated murine Lyme arthritis and carditis through the induction of tolerance. PLoS One 2011; 6(2): e17414.

66. Hedl M, et al. Chronic stimulation of Nod 2 mediates tolerance to bacterial products. Proc Natl Acad Sci U S A 2007; 104(49): 19440-5.

67. Kullberg BJ, et al. Crohn's disease patients homozygous for the 3020insC NOD2 mutation have a defective NOD2/TLR4 cross-tolerance to intestinal stimuli. Immunology 2008; 123(4): 600-5.

68. Watanabe T, et al. NOD2 is a negative regulator of Toll-like receptor 2-mediated T helper type 1 responses. Nature immunology 2004; 5(8): 800-8.

69. Shaw MH, et al. The ever-expanding function of NOD2: autophagy, viral recognition, and T cell activation. Trends in immunology 2011; 32(2): 73-9.

70. Seder RA, et al. CD28-mediated costimulation of interleukin 2 (IL-2) production plays a critical role in T cell priming for IL-4 and interferon gamma production. $\mathrm{J}$ Exp Med 1994; 179(1): 299-304.

71. Barreau F, et al. Nod2 regulates the host response towards microflora by modulating $\mathrm{T}$ cell function and epithelial permeability in mouse Peyer's patches. Gut 2010; 59(2): 207-17.

72. Sartor RB. Key questions to guide a better understanding of host-commensal microbiota interactions in intestinal inflammation. Mucosal immunology 2011; 4(2): 127-32.

73. Chassaing B, Darfeuille-Michaud A. The commensal microbiota and enteropathogens in the pathogenesis of inflammatory bowel diseases. Gastroenterology 2011 140(6): 1720-28.

74. Willing BP, et al. A pyrosequencing study in twins shows that gastrointestinal microbial profiles vary with inflammatory bowel disease phenotypes. Gastroenterology 2010; 139(6): 1844-1854 e1.

75. Maslowski KM, Mackay CR. Diet, gut microbiota and immune responses. Nat Immunol 2011; 12(1): 5-9.

76. Atarashi K, et al. Induction of colonic regulatory $\mathrm{T}$ cells by indigenous Clostridium species. Science 2011; 331(6015): 337-41.

77. Sokol H, et al. Faecalibacterium prausnitzii is an anti-inflammatory commensal bacterium identified by gut microbiota analysis of Crohn disease patients. Proceedings of the National Academy of Sciences of the United States of America 2008; 105(43): 16731-6.

78. Darfeuille-Michaud A, et al. High prevalence of adherent-invasive Escherichia col associated with ileal mucosa in Crohn's disease. Gastroenterology 2004; 127(2): 412-21.

79. Kotlowski R, et al. High prevalence of Escherichia coli belonging to the B2+D phylogenetic group in inflammatory bowel disease. Gut 2007; 56(5): 669-75.

80. Barnich N, et al. CEACAM6 acts as a receptor for adherent-invasive E. coli, supporting ileal mucosa colonization in Crohn disease. The Journal of clinical investigation 2007; 117(6): 1566-74

81. Rolhion N, et al. Abnormally expressed ER stress response chaperone Gp96 in CD favours adherent-invasive Escherichia coli invasion. Gut 2010; 59(10): 1355-62.

82. Eaves-Pyles T, et al. Escherichia coli isolated from a Crohn's disease patient adheres, invades, and induces inflammatory responses in polarized intestinal epithelial cells. Int J Med Microbiol 2008; 298(5-6): 397-409.

83. Chassaing B, et al. Crohn disease - associated adherent-invasive E. coli bacteria target mouse and human Peyer's patches via long polar fimbriae. The Journal of clinical investigation 2011; 121(3): 966-75.

84. Glasser AL, et al. Adherent invasive Escherichia coli strains from patients with Crohn's disease survive and replicate within macrophages without inducing host cell death. Infection and immunity 2001; 69(9): 5529-37.

85. Meconi S, et al. Adherent-invasive Escherichia coli isolated from Crohn's disease patients induce granulomas in vitro. Cellular microbiology 2007; 9(5): 1252-61.

86. Glimcher LH. Trawling for treasure: tales of T-bet. Nature immunology 2007 8(5): 448-50.

87. Stucchi A, et al. A new transcription factor that regulates TNF-alpha gene expression, LITAF, is increased in intestinal tissues from patients with CD and UC. Inflammatory bowel diseases 2006; 12(7): 581-7.

88. Baker PI, Love DR, Ferguson LR. Role of gut microbiota in Crohn's disease. Expert Rev Gastroenterol Hepatol 2009; 3(5): 535-46.

89. Neurath MF Finotto S. IL-6 signaling in autoimmunity, chronic inflammation and inflammation-associated cancer. Cytokine Growth Factor Rev 2011; 22(2): 83-9.

90. Lee YK, et al. Developmental plasticity of Th17 and Treg cells. Current opinion in immunology 2009; 21(3): 274-80. 
91. Kamada N, et al. Unique CD14 intestinal macrophages contribute to the pathogenesis of Crohn disease via IL-23/IFN-gamma axis. The Journal of clinical investigation 2008; 118(6): 2269-80.

92. Ahern PP, et al. Interleukin-23 drives intestinal inflammation through direct activity on T cells. Immunity 2010; 33(2): 279-88.

93. Maloy KJ, Kullberg MC. IL-23 and Th17 cytokines in intestinal homeostasis. Mucosal immunology 2008; 1(5): 339-49.

94. Leppkes M, et al. RORgamma-expressing Th17 cells induce murine chronic intestinal inflammation via redundant effects of IL-17A and IL-17F. Gastroenterology 2009; 136(1): 257-67.
95. Sonnenberg GF, et al. Pathological versus protective functions of IL-22 in airway inflammation are regulated by IL-17A. J Exp Med 2010; 207(6): 1293-305.

96. Sonnenberg GF, Fouser LA, Artis D. Border patrol: regulation of immunity, inflammation and tissue homeostasis at barrier surfaces by IL-22. Nat Immunol 2011; 12(5): 383-90.

97. Wolk K, et al. Biology of interleukin-22. Semin Immunopathol 2010; 32(1): 17-31.

98. Strober W, Fuss IJ. Proinflammatory cytokines in the pathogenesis of inflammatory bowel diseases. Gastroenterology 2011; 140(6): 1756-67.

99. Smith PD. Principles of Mucosal Immunology, Garland Science, Taylor \& Francis Groupe, LLC 2013: 529.

Received: $30 / 04 / 2014$

Accepted in revised form: 08/09/2014

\section{Corresponding author:}

Tomáš Hrnčír, M.D., Ph.D., Department of Immunology and Gnotobiology, Institute of Microbiology, Academy of Sciences of the Czech Republic, 54922 Nový Hrádek, Czech Republic; e-mail: tomas@gnotolab.com 